\title{
Investigación \\ Oferta formativa, mercado laboral y perfiles profesionales
}

Pau Rausell Köster' > Francesc Cabañés Martinez" > Ximo Revert Roldán"'

\section{Resumen}

Los autores analizan, en primer lugar, el perfil profesional de los gestores de patrimonio cultural, por considerar que, dadas las caracteristicas de la actividad de la gestión patrimonial en España y las actuales discusiones sobre la inserción universitaria de la gestión cultural, se trata de un caso paradigmático de las dificultades que entraña definir en nuestro pais la oferta formativa, el mercado laboral y los perfiles profesionales vinculados al sector cultural.

En segundo término, y siguiendo el modelo de las tres categorías aportado por X. Greffe, tratan de acercarse a la estructura y composición porcentual del mercado laboral ligado al patrimonio en España. La principal dificultad para esta tarea radica en la práctica ausencia de datos e investigaciones fiables. Seguidamente se apuntan algunas posibilidades de crecimiento en la ocupación, muy relacionadas con las políticas públicas y el turismo cultural.

Finalmente, repasan las caracteristicas de la oferta formativa en el ámbito de la gestión patrimonial.

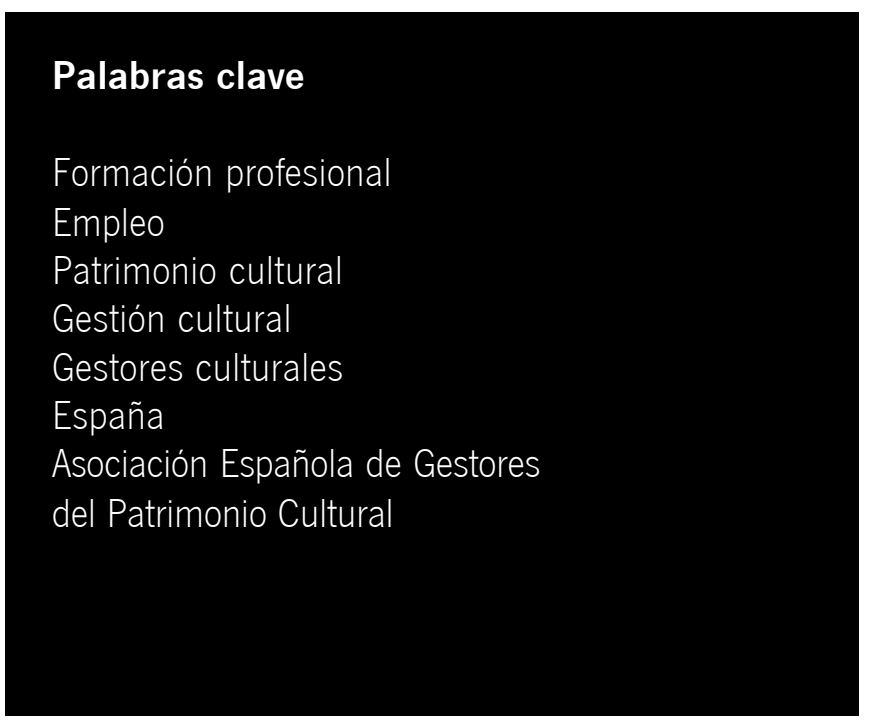

\section{Introducción}

Desde hace algunos años, la gestión del patrimonio se ha convertido en un sector recurrente en la literatura política y académica que habla sobre los nuevos nichos emergentes de ocupación. Ya en 1992 la Tercera Conferencia Europea de los Ministros responsables de la protección del patrimonio apuntaba, en su análisis anticipatorio ${ }^{1}$, que había que explorar las conexiones entre los aspectos sociales de la preservación del Patrimonio y las propias políticas de empleo, por lo que implícitamente intuían unas grandes expectativas sobre la posibilidades de generar ocupación en el ámbito de la gestión del patrimonio. Y en la misma línea insistía el Informe Delors del año siguiente.

Los resultados en España, a falta de estudios e investigaciones más detalladas, parecen ser más modestos de lo esperado. Aspecto que desde nuestro punto de vista evidencia más un excesivo entusiasmo inicial que algún problema estructural en el mercado laboral de valorización del patrimonio. Lo que destacamos a modo de pinceladas en el presente texto es que si, por una parte, ya resulta un ejercicio conceptualmente complicado definir en la práctica qué es el mercado laboral de las actividades ligadas a la producción, conservación, difusión y distribución del patrimonio, por otra, la inmadurez en la definición del perfil profesional así como la dispersión y confusión de la oferta formativa, nos sitúan ante una tesitura que reclama sin lugar a dudas una mayor profundidad en la aproximación conceptual y mucha, mucha más investigación.

Estas consideraciones, que podrian ser aplicables a cualquiera de las actividades ligadas a la cultura, resultan especialmente pertinentes en al ámbito de la gestión patrimonial. Y son pertinentes tanto por la características de la propia actividad de la gestión patrimonial y su importancia sobre la estructura de las actividades culturales en España, como por las recientes discusiones alrededor de la inserción de la gestión cultural en la formación reglada, tal y como apunta la enésima reforma del modelo universitario, en proceso de convergencia europea. Las limitaciones del espacio, así como las capacidades de los autores, sin embargo aconsejaban abandonar la pretensión de realizar un análisis comparado sobre la realidad europea. Una tarea ineludible que presenta en este mismo monográfico tal y como algunas aportaciones de gran nivel.

\section{Los perfiles profesionales del patrimonio, espacios comunes y diferenciados de la gestión cultural}

La generalización e implantación de las políticas culturales y su gestión no sólo han servido para dotar a la población de más y 
" Gestor Cultural. Director del Museo Taurino de la Diputación de Valencia

III Técnico en Cultura. Profesor del Máster de Conservación y Gestión del Patrimonio Cultural de la Universidad de Valencia mejores servicios culturales, sino que también ha contribuido a incrementar la conceptualización de las acciones y la fundamentación teórica de las prácticas. Gracias a este proceso de retroalimentación mutua hoy podemos avanzar en la delimitación, entre otros, de un perfil profesional alrededor de la cultura diferenciado de las tradicionales profesiones culturales. Así, de acuerdo con una clasificación que va ampliando su aceptación, podemos profundizar en algunas dimensiones que nos permiten una mayor precisión conceptual.

La gestión de la cultura puede ser concebida desde su doble faceta de administración e implementación, pública o privada, de intervenciones sobre el ámbito de la cultura, y por tanto asimilable a cualquier otro tipo de administración (de la sanidad, de la educación o de lo social, por poner diferentes ejemplos en el área de los servicios personales). Nuestro objetivo es situar el perfil profesional del gestor del patrimonio cultural dentro de este gran panorama de profesiones incluidas en la gestión de la cultura.

El gestor cultural es un profesional que desarrolla tareas relacionadas con la promoción y difusión de la cultura (creación, producción, distribución, consumo cultural y conservación), teniendo bien clara la distancia que los separa de las actividades estrictamente creativas. En este sentido es oportuno destacar que estas tareas son culturales pero están separadas de la creación cultural (APGCC, 1996). Dicho de otro modo, el gestor cultural no pinta, no actúa, no interpreta, no restaura o excava un yacimiento, su labor se centra en la socialización de las creaciones de estos profesionales.

Por otro lado, el gestor cultural desarrolla tareas de análisis y diseño de planes y programas culturales, esto es: planificación, gestión de programas y administración de recursos y su evaluación. Esta circunstancia lo diferencia claramente de otros perfiles. Tampoco son, por tanto, ni taquilleros, maquinistas, regidores o cajeros de superficies culturales, etc.

Con estas premisas, la gestión y, por tanto, la profesión de gestor cultural se presenta como una actividad profesional que se puede posicionar en referencia a tres ejes principales:

$>$ Opera en el sector cultural. El gestor cultural manipula, articula, transforma, valoriza bienes y servicios ligados a la dimensión simbólica de un grupo social, es decir de sus referentes culturales. Aunque la definición de la dimensión simbólica no es un concepto delimitado, ni necesariamente constante en término temporales y geográficos, podemos aproximarnos a él a través de un listado que contendría de manera precisa actividades ligadas a la artes escénicas, plásticas, las industrias culturales, el patrimonio material e inmaterial y sus formas de difusión, la música y la artesanía, y de forma menos precisa a otros ámbitos como el turismo cultural, el diseño, la moda, la arquitectura etc.

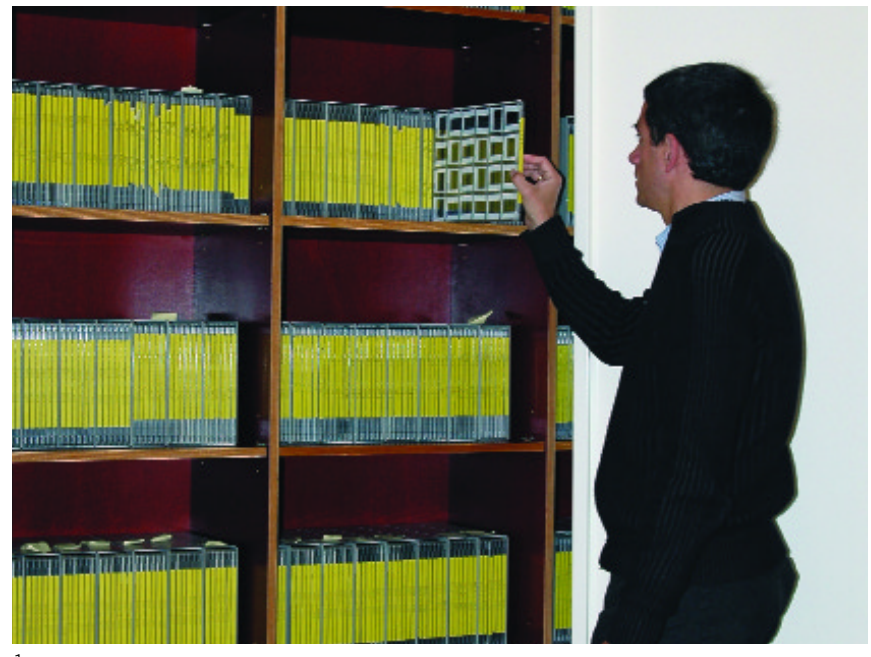

1

$>$ Gestiona. En este eje se incide sobre los procesos de gestión necesarios para la puesta en marcha de las acciones y programas, desde la planificación estratégica, el marketing, la gerencia administrativa y presupuestaria, la gestión de personal, la gestión y planificación de programas y equipamientos o la evaluación.

$>$ Actúa en distintas fases del proceso del producto. Este tercer eje delimita el momento de la participación del gestor cultural en la fase del producto cultural. Asi se podrian clasificar a los gestores culturales en función de si su intervención se realiza en la fase de la información/formación, creación, producción, distribución, consumo, o conservación del bien o servicio cultural

A medida que van madurando la actividades relacionadas con la gestión cultural, la combinación de los tres ejes configura un volumen de perfiles profesionales que muestran procesos de consolidación y especialización con relativa autonomía. No obstante, la gestión y administración y todas sus funciones anexas son necesariamente comunes, como también lo son aquéllas que tienen que ver con la faceta de promoción y difusión del elemento cultural. El sector patrimonial no es una excepción.

La Asociación Española de Gestores de Patrimonio Cultural ubica, bajo nuestro punto de vista, a estos profesionales dentro del marco común de la gestión cultural sin renunciar a establecer sus propias particularidades del sector: "El gestor del patrimonio cultural no es un artista, ni un conservador de museos, ni un arqueólogo, ni un historiador del arte, ni un restaurador ni un arquitecto, aunque pueda provenir de cualquiera de estas profesiones (...) El gestor de patrimonio cultural es eminentemente un administrador de recursos, y su formación ampliamente multidisciplinar requiere un amplio y exhaustivo conocimiento específico acerca del elemento del Patrimonio Cultural que haya de gestionar, y además, múltiples y variados conocimientos que incluyen, 


\section{Investigación}

Oferta formativa, mercado

laboral y perfiles

profesionales

\section{Pau Rausell Köster} y otros
Tabla 2. Fuente: Elaboración propia a partir de Ministerio de Cultura (2002): Las cifras de la cultura en España

\section{Tabla 1}

Nivel 1. Empleos directos

Nivel 2. Empleos indirectos en trabajos

de conservación y restauración

Nivel 3. Empleos inducidos
4\% Monumentos

8\% Patrimonio en sentido amplio
Empleos vinculados a los servicios de acogida en los elementos patrimoniales (monumentos y patrimonio) y que básicamente permiten el consumo final.
$7,8 \%$

Empleos que no están vinculados permanentemente a un determinado emplazamiento patrimonial, sino que realizan y producen bienes y servicios destinados de demanda intermedia.
$32 \%$ Turismo

$47 \%$ Resto sectores
Empleos derivados de la existencia del patrimonio como mecanismo generador de flujos turísticos o como intermediarios entre la oferta patrimonial y la demanda:

$>$ Sector turístico

$>$ Sector de las artes y las industrias culturales

$>$ Otros sectores

\section{Tabla 2}

Miles de euros

Gasto de la Administración General del Estado, por programas 2000 (Presupuesto Inicial)

Protección y Administración del Patrimonio Histórico Nacional

Conservación y Restauración de Bienes Culturales

Gasto Público por programas de las CC.AA. 2000 (Presupuesto Inicial)

Patrimonio histórico y artístico

$141 \%$

Gasto de las Corporaciones Locales, según capítulos y programas 1996 (Presupuesto liquidado)

Arqueología y protección del patrimonio histórico-artístico

Total $\quad 491.033$
Tasa de

crecimiento

$1991-2000$

(euros corrientes)
298.547

64.957

92.412

35.117
$61 \%$

$13 \%$

n.d.

$42 \%$

$100 \%$ entre otros, desde las técnicas de administración de empresas, dirección de recursos humanos al marketing cultural. En conseguir hacer compatible la conservación del bien cultural que se le haya encomendado con la obtención de una rentabilidad social, cultural, económica del mismo".

Desde el punto de vista de la consolidación profesional, cabe decir que dada la mayor proyección temporal de las políticas de protección del patrimonio, y su claro acento en la dimensión técnica de la misma, los gestores del patrimonio, por un parte, muestran unos signos más evidentes de consolidación profesional, pero al mismo tiempo una mayor rigidez en su praxis dado su origen en los aspectos más técnicos ligados a la conservación y preservación. En consecuencia, visibilizan una mayor resistencia a interiorizar plenamente una definición más pluridisciplinar.

Establecida la relación de esta "profesión" con su tronco común, sólo queda significar la paulatina especialización que el en futuro presentará este sector de la profesión y la necesidad de aumentar lo conocimientos específicos necesarios para desarrollar el trabajo. Cuestión que por otro lado también afectará a los demás sectores y que se produce como consecuencia del crecimiento de la oferta y servicios culturales, tanto en su dimensión cuantitativa como cualitativa, al conformar propuestas más sofisticadas y complejas desde el punto de vista conceptual y organizativo. 
2. Documentalistas. Imagen: Javier Romero. Fototeca del IAPH

3. Restauradora de pintura. Imagen: Eugenio Fernández. IAPH
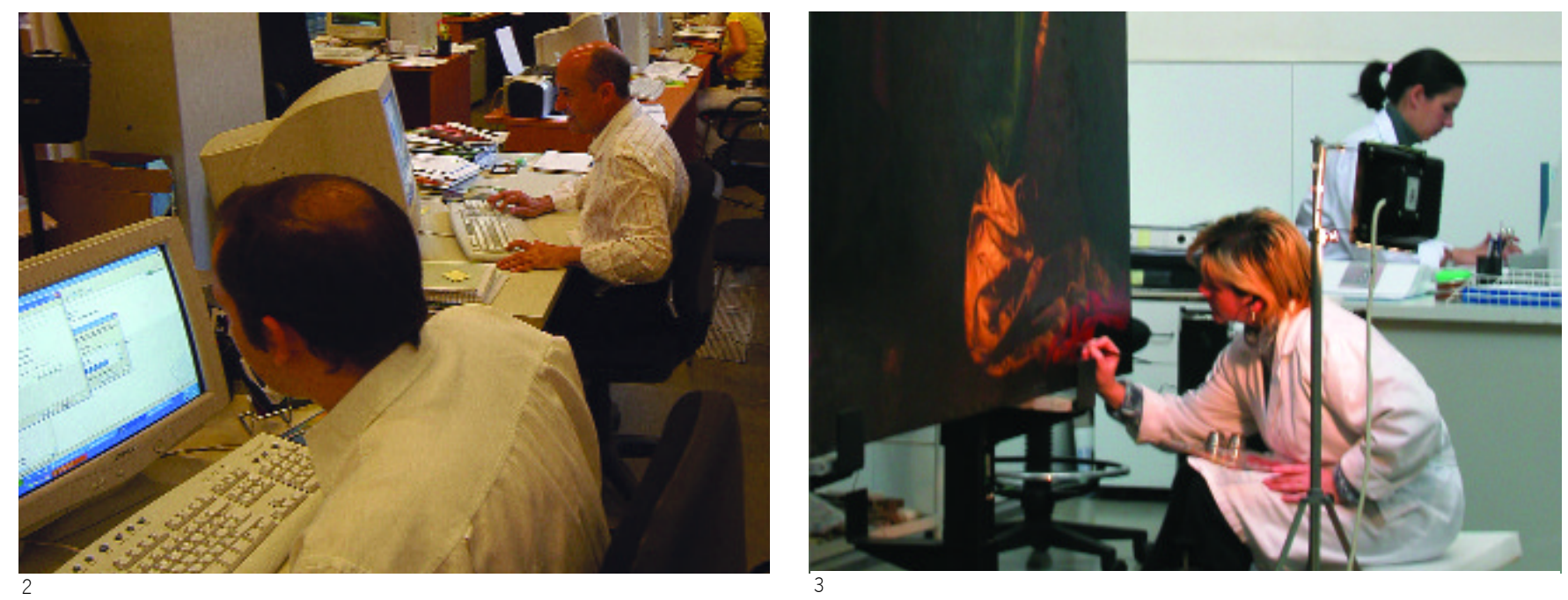

\section{El mercado laboral del patrimonio. Algunas consideraciones sobre dimensiones y tendencias}

Xavier Greffe (Greffe, X., 2003) utiliza unas categorias útiles para la demarcación de un mercado laboral ligado al patrimonio, ya que nos permite visualizar con bastante claridad los distintos niveles de vinculación entre el patrimonio y el empleo (tabla 1). El primer círculo de empleos ligados al patrimonio lo ubica en los empleos vinculados a los servicios de acogida en los elementos patrimoniales (monumentos y patrimonio ${ }^{2}$ ). Se trata de empleos públicos y privados que contribuyen a que los espacios del patrimonio estén abiertos y por tanto valorizados. Las características de estos empleos es que se ubican en el espacio de los bienes patrimoniales y posibilitan el consumo final. El segundo nivel de ocupación lo sitúa en aquellos trabajos de conservación y restauración no contabilizados en el nivel anterior, es decir que no están vinculados permanentemente a un determinado emplazamiento patrimonial, sino que realizan y producen bienes y servicios destinados de demanda intermedia (empresas de restauración, centros públicos de restauración que proveen servicios a distintos espacios patrimoniales, artesanos, etc.). El siguiente nivel corresponde a aquellas ocupaciones derivadas de la existencia del patrimonio como mecanismo generador de flujos turisticos o como intermediarios entre la oferta patrimonial y la demanda, y entre éstos cabría distinguir entre los estrictamente vinculados al sector turístico, aquéllos que afectan al sector de las artes y las industrias culturales y, finalmente, aquéllos vinculados a otras actividades no culturales.

Esta estructura y su composición porcentual en Francia nos permite realizar alguna atrevida estimación sobre la realidad española, que sólo puede ser entendida como punto de referencia ini- cial para motivar futuras investigaciones. El único dato con ciertas garantías con el que contamos (que a nosotros nos conste) sobre el empleo en el sector del patrimonio es el que nos ofrece el equipo de Isabel García (Garcia, I., Fernández, Y., Zofio, J.L, 2003). El empleo ligado al patrimonio histórico era en el año 1997 de $7.617^{3}$ ocupados. Con este dato, suponiendo una estructura del empleo directo e inducido similar a la de Francia y extrapolando una tasa de crecimiento del empleo del 3,3\% en los sectores culturales de actividades de bibliotecas, archivos, museos y otras instituciones culturales, desde 1994 al 2000 (tal como se puede derivar de Las cifras de la Cultura en España), podríamos aventurar, muy temerariamente, que en el año 2005 los empleos totales en España ligados al patrimonio se situarían en una horquilla entre los 85.000 y los 160.000 trabajadores. De los cuales una tercera parte correspondería al sector turístico, y casi el $50 \%$ se ubicaría en el resto de los sectores (incluidos los sectores e industrias culturales), y finalmente, aproximadamente una quinta parte, a actividades profesionales de valorización, difusión y conservación del patrimonio. Este último segmento que podría moverse entre los 17.000 y los 32.000 ocupados podría definir el núcleo duro del perfil profesional que buscamos.

Parece claro que lo que constituye el grueso del perfil profesional del gestor cultural, con habilidades específicas en la interpretación, valorización, difusión y gestión del patrimonio, habria que ubicarlo en esa franja difusa de esa última quinta parte, de la cual tendríamos que excluir tanto al personal de acogida auxiliar (desde bedeles a guardas de seguridad o administrativos) como a aquéllos dedicados exclusivamente a trabajos técnicos de restauración y conservación sin funciones de interpretación y valorización.

De todas formas, las intuiciones que nos revelan la estructura de la ocupación del patrimonio nos pueden inducir también a algunas consideraciones sobre las posibilidades de crecimiento 


\begin{tabular}{l}
\hline Investigación \\
Oferta formativa, mercado \\
laboral y perfiles \\
profesionales \\
Pau Rausell Köster \\
y otros
\end{tabular}

de la ocupación. Parece claro que la dinámica de la ocupación en el sector del patrimonio puede venir condicionada por dos vectores principales y uno más secundario. El primer vector principal reside en la dimensión, magnitud y crecimiento de las políticas públicas referidas al patrimonio. No nos cabe ninguna duda que el principal agente valorizador del patrimonio en España es el sector público y especialmente las administraciones autonómicas como podemos observar en la tabla 2. En ellas se puede observar que las CC.AA. juegan un papel determinante tanto en términos de dimensiones absolutas (suponían más del $60 \%$ del gasto público total) como de crecimiento durante la década de los 90.

Las estrategias concretas de las CC.AA. sobre las politicas de protección del patrimonio devienen así en elementos centrales para explicar las dinámicas de crecimiento de la ocupación en el sector ${ }^{4}$.

El segundo vector relevante para la evolución de la ocupación en el sector del patrimonio es el desarrollo del turismo cultural. Como hemos comprobado en los datos anteriores, el turismo cultural es una de las dimensiones de mayor impacto en el ámbito de la creación de empleo ligado al patrimonio. Resulta indiscutible que el turismo cultural es una actividad emergente en España y que dados sus determinantes desde el punto de vista de la demanda es previsible que estas circunstancias persistan a medio y largo plazo. Estas tendencias van a tener un efecto sobre las capacitaciones profesionales exigidas de forma que sin duda se incrementará la sofisticación de las herramientas, ya que empezamos a establecer marcos donde los procesos de valorización del patrimonio no sólo han de ser eficaces desde el punto de vista instrumental sino competitivos en un entorno donde se multiplican las ofertas. Esta circunstancia empuja también a las profesiones de gestión y valorización del patrimonio hacia una creciente complejidad.

Finalmente, el tercer vector dinamizador del mercado de trabajo, quizás de menor impacto, resulta ser la propia presión que ejercen las propuestas formativas en el ámbito de la gestión cultural del patrimonio. En este sentido, la oferta formativa no sólo funciona como estrategia reactiva a las demandas del mercado, sino que se origina por lógicas diversas y los "formados" constituyen un elemento capaz de generar su propia demanda, cumpliendo la Ley de Say ${ }^{5}$. Esta circunstancia puede ser especialmente relevante en aquellas Comunidades Autónomas de dimensiones reducidas y con mercados de trabajo relacionados con la gestión cultural muy estrechos pero con ofertas formativas dinámicas. El modelo formativo, por tanto, no va a ser neutral.

La concurrencia de estos tres vectores va a determinar la articulación de los mercados de trabajo ligados al patrimonio en distintos entornos territoriales y de su presiones relativas dependerá la configuración concreta de las características del marco laboral donde se muevan los gestores del patrimonio.
4. Arqueólogas subacuáticas. Imagen: Fondo gráfico del Centro de Arqueología Subacuática (IAPH)

\section{Las características de la oferta formativa en el ámbito de la gestión patrimonial}

No cabe ninguna duda que dentro del abanico de oferta en formación en gestión cultural es perceptible un claro predominio en el ámbito del patrimonio (Martinell, 2001).

En primer lugar cabe destacar que, territorialmente, en España la oferta formativa está bien distribuida correspondiendo los programas formativos más desarrollados, pluridisciplinares y de larga duración a las comunidades autónomas con mayor proyección y desarrollo de sus políticas culturales en materia de patrimonio: Andalucia, Castilla y León, Cataluña, País Valenciano, Madrid, País Vasco y Galicia.

Las ofertas más fundamentadas tienen en común programas con contenidos y materias similares, aunque la estructura y metodología para formalizar cada oferta sea diversa. En algunos cursos los contenidos se estructuran en torno a análisis, conservación, difusión y gestión de patrimonio, y en otros casos se estructura en torno a ámbitos disciplinares como lo social, lo económico, lo jurídico y lo cultural.

En los últimos cursos, sin embargo, se observa el agotamiento de las propuestas más generalistas y una proliferación de ofertas de media y corta duración más monográficas e instrumentales.

Genéricamente podemos perfilar el carácter de la formación en materia de patrimonio cultural a tenor de, al menos, cuatro enfoques bien marcados que vienen a definir los rasgos de cada oferta formativa según entiendan el patrimonio cultural como objeto, como sujeto, como contexto o como discurso.

$>$ El patrimonio cultural como objeto: define aquellas ofertas formativas vinculadas a la conservación o restauración de patrimonio. Suelen provenir de disciplinas técnicas (arquitectura, bellas artes...).

$>$ El patrimonio cultural como sujeto: éste se perfila como eje de políticas públicas activas y específicas, como recurso de desarrollo, como expresión cultural sobre la que se ciernen derechos, obligaciones, o valores y es así mismo soporte de proyectos o planificaciones en torno al cual giran o se predefinen otras políticas.

$>$ El patrimonio cultural como contexto: nos referimos a aquellas ofertas donde el patrimonio forma parte o contribuye a la especialización en gestión cultural desde parámetros más generalistas, como un apéndice más de las políticas culturales o las políticas de desarrollo territorial (comercio, turismo, sostenibilidad). Quizá esta oferta formativa sea la más instrumental.

> Patrimonio y discurso: existen ofertas que tratan el patrimonio como vertebrador de un discurso de reevaluación identitaria, 


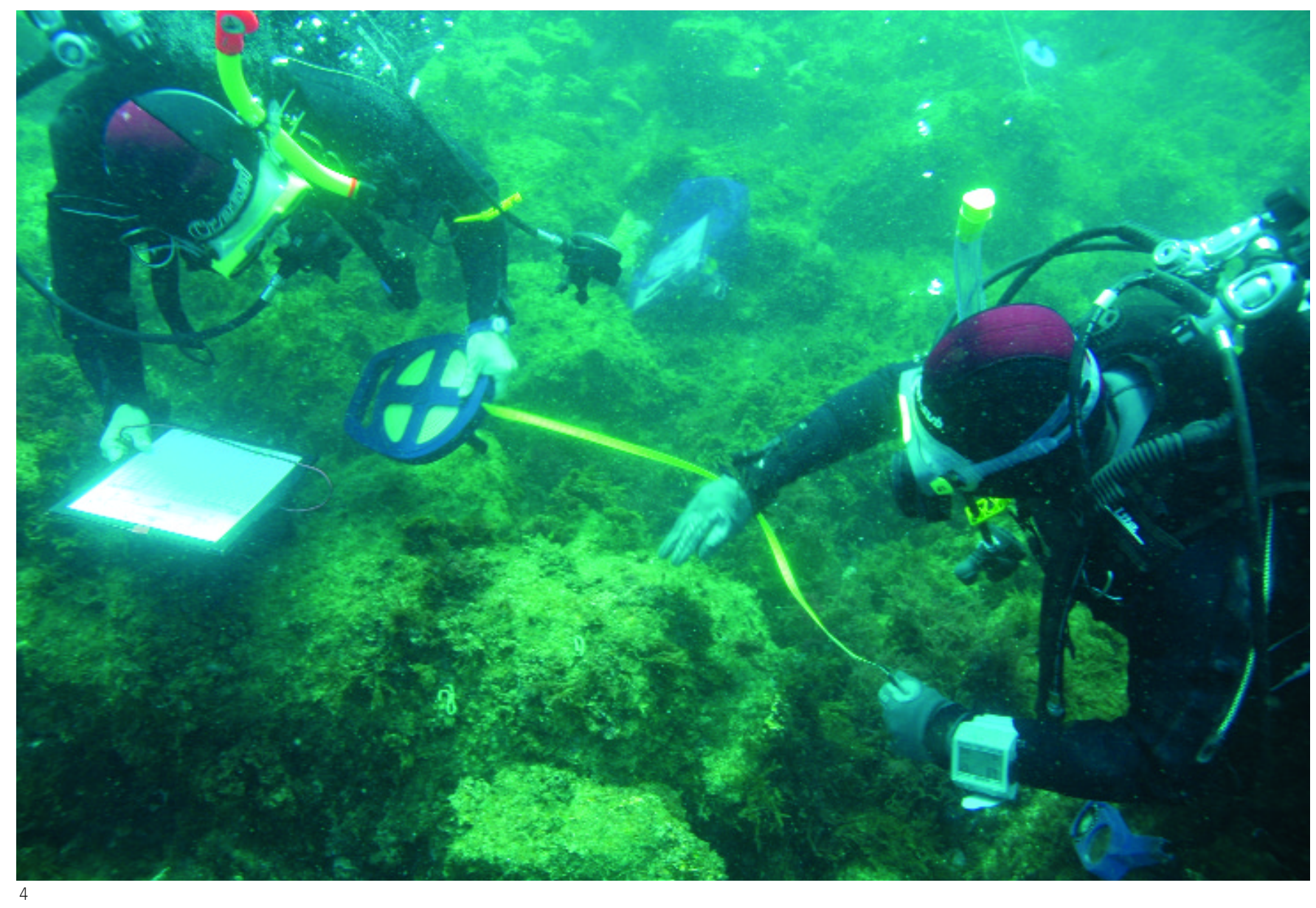

como argumento para generar itinerarios, o nuevos productos culturales, como soporte al desarrollo social de una comunidad, o como profundización en el autorreconocimiento del propio territorio. Estas ofertas incluyen en su programación de contenidos una dosis importante de referencias a casos concretos, accediendo a lo general desde lo particular.

En general podriamos afirmar que en aquellas CC.AA. con numeroso patrimonio histórico más convencional (principalmente patrimonio inmueble) y políticas poco activas en el ámbito de la reafirmación identitaria predominará las ofertas del primer tipo (patrimonio como objeto y sujeto), donde aparecen acentuados los módulos y contenidos sobre la gestión de patrimonio como un procedimiento en si mismo que garantice la conservación y su durabilidad. Mientras que en aquellas CC.AA. con perfil más identitario se decantarán hacia el reforzamiento del papel del contexto y del discurso (especialmente en las denominadas nacionalidades históricas) para instalarlo en un proyecto de gestión de recursos para el territorio

Mayoritariamente el origen del profesorado que imparte los contenidos de esta oferta formativa es natural del territorio o de la universidad que lanza la oferta, aunque la tendencia es a incorporar cada vez más, y sobre todo en los másters o posgrados pioneros, a profesorado externo, pocas veces internacional o extranjero. El perfil profesional de este profesorado es mayoritariamente académico y disciplinariamente asociado a la historia del arte, a la historia, a la arquitectura y el urbanismo, también al derecho y a la economía. Más circunstancial resulta la presencia de sociólogos, arqueólogos, psicólogos sociales, o científicos e ingenieros.
Externos al mundo académico, en torno a una quinta parte del profesorado, la componen propiamente profesionales de diversos sectores de la cultura, políticos con directa responsabilidad en gestión y administración de departamentos públicos, funcionarios al frente de grandes eventos, reduciéndose la participación de otros agentes sociales (empresa, banca, fundaciones, asociaciones, ...) a pequeños módulos, seminarios o ciclos de conferencias.

Sin lugar a dudas, la asunción de los gobiernos autónomos de competencias en materia de cultura ha elevado la necesidad de profesionales primero de la gestión cultural generalista (años ochenta y noventa), para pasar a demandar gestores o agentes dinamizadores de patrimonio cultural con sus especificidades y singularidades profesionales. Quizá esta demanda formativa y profesional ha incentivado que muchas disciplinas orienten sus investigaciones y promuevan sus tesis hacia enfoques que contengan el patrimonio cultural como objeto o sujeto facilitando que muchos estudiosos o titulados en disciplinas tradicionales se incorporen al mercado formativo o profesional socializando así sus perspectivas y conocimientos.

Estamos, por tanto, ante un panorama formativo que va completando, a partir de una estrategia de "mancha de aceite", la incorporación de disciplinas diversas para contextualizar al patrimonio en su necesaria dimensión integral, transversal y multidisciplinar.

\section{A modo de conclusiones}

En general, podemos apreciar que nos vamos ubicando en una sociedad que quizás ha superado claramente una primera fase 


\section{Investigación}

Oferta formativa, mercado

laboral y perfiles

profesionales

\section{Pau Rausell Köster}

y otros
5. Técnico de difusión. Imagen: Dolores Lobillo

aporten una visión mucho más nítida y precisa de la realidad de la profesión, su conceptualización y su medida.

\section{Notas}

${ }^{1}$...to explore the social aspects of conservation more fully by integrating them with housing and employment policies. Resolution No. 3 on the priorities of a Pan-european cultural heritage project.

${ }^{2}$ Greffe realiza una doble distinción entre "Monumentos" que corresponde a los monumentos, sitios arqueológicos y otros espacios protegidos por las leyes de patrimonio, mientras que se refiere a "Patrimonio" cuando habla de los empleos ligados a los museos o a otras formas de patrimonio (Greffe, 2003:31).

${ }^{3}$ Que correpondería al nivel 1 de las categorias de Greffe, referido sólo a los monumentos y a parte del nivel 2 de conservación y preservación.

${ }^{4}$ En el año 2000, las CC.AA. en conjunto dedicaban un 34\% de su gasto en cultura al Patrimonio Histórico y Artístico, aunque la varianza respecto a esta cifra media es bastante elevada. Por ejemplo, la Comunidad Valenciana en el período 2002-2005 sólo dedicaba un $13 \%$ a esas partidas (Rausell, 2005)

${ }^{5}$ Frecuentemente la ley de Say se resume en la expresión simplificada de que la oferta crea su propia demanda.

\section{Bibliografía}

ALONSO, J.A.; SANZ, M. (2000) El Patrimonio histórico de España desde una perspectiva económica: una primera aproximación al gasto en conservación, restaut ración y rehabilitación del patrimonio histórico español. Madrid: Fundación Caja Madrid, 2000

ASOCIACIÓN DE PROFESIONALES DE LA GESTIÓN CULTURAL DE CATALUÑA (1996) La gestión Cultural: una Nueva Profesión a debate. Barcelona: APGCC, 1996

GARCÍA, I.; FERNÁNDEZ, Y.; ZOFIO, J.L. (2003) The economic Dimension of the Culture and Leisure Industry in Spain: National, Sectorial and regional Analysis. Journal of Cultural Economics, v. 27, n²1, 2003, pp. 9-30

GREFFE, X. (2003) "La valorisation économique du parimoine". La Documentation Française. París: 2003

INCANOP (1997) Les professions de la cultura. Barcelona: Generalitat de Catalunya, 1997

INTERARTS (2000) Proyecto: FORMAT Gestión Cultural: Formación y Empleo. Una apuesta hacia su Internacionalización. Barcelona: Fundación Interarts, 2000

MARTINELL, A. (2001) La gestión cultural: singularidad profesional y perspectivas de futuro (recopilación de textos) www.iberformat.org/AMartinell.pdf (consulta: abril de 2005)

MINISTERIO DE CULTURA (2002) Las cifras de la Cultura en España. Estadisticas e indicadores. Madrid: Ministerio de Cultura, 2002

RAUSELL, P. (2005) Polítiques culturals valencianes: patrimoni, recursos i participació ciutadana. Conferencia presentada en el II Congrés de Patrimoni Cultural Valencia. Sagunto, febrero de 2005 